\title{
Acceptability of the method of administration of a patient-reported outcome measure (PROM) with stroke survivors, a randomised controlled trial protocol
}

Alexander Smith ${ }^{1}$, Anna Pennington ${ }^{1}$, Ben Carter ${ }^{2}$, Stephanie Gething ${ }^{1}$, Michelle Price ${ }^{3}$, James White ${ }^{4}$, Richard Dewar ${ }^{4}$ and Jonathan Hewitt ${ }^{1,5^{*}}$

\begin{abstract}
Background: UK-wide national clinical guidelines promote routine 6-month post-stroke follow-up assessment. However, as part of this 6-month assessment little information is gathered from the patient's perspective. The means of collecting this patient-centred information might be served best by a patient-reported outcome measure (PROM) at the 6-month assessment time point. Currently, four different methods of 6-month follow-up assessment occur; the most common being face-to-face interview followed by telephone interview, postal questionnaire and online questionnaire. Therefore, this study will investigate if the acceptability of telephone, online or postal administration of a PROM at the 6-month post-stoke time point is not inferior to face-to-face administration.

Methods/design: A UK multicentre, blinded (analyst and researcher), pragmatic, non-inferiority study, with 80\% power using a $2.5 \%$ non-inferiority margin was designed to compare the acceptability of three modes of administration (telephone interview, postal questionnaire and online questionnaire) compared with face-to-face interview administration of a PROM. We plan to approach and randomise a minimum of 808 potentially eligible participants, 202 participants per group.

Discussion: The aim of this ongoing research is to understand if there is a difference between face-to-face administration and the other three methods of administering a PROM as a patient-centred supplement to the 6-month review for stroke survivors. In utilising a pragmatic design, it is believed that this study will offer UK wide generalisable results, of the acceptability of the methods under investigation, to inform clinicians and commissioners of stroke services.
\end{abstract}

Trials registration: ClinicalTrials.gov: NCT03177161. Registered on 6 June 2017.

Keywords: Stroke, CVA, PROM, Questionnaire, Response rate, Online, Face-to-face, Postal, Telephone, Non-inferiority

\footnotetext{
*Correspondence: HewittJ2@cardiff.ac.uk

${ }^{1}$ Aneurin Bevan University Health Board, South Wales, UK

${ }^{5}$ Division of Population Medicine, Cardiff University, Cardiff, UK

Full list of author information is available at the end of the article
}

(c) The Author(s). 2018 Open Access This article is distributed under the terms of the Creative Commons Attribution 4.0 International License (http://creativecommons.org/licenses/by/4.0/) which permits unrestricted use, distribution, and reproduction in any medium, provided you give appropriate credit to the original author(s) and the source, provide a link to the Creative Commons license, and indicate if changes were made. The Creative Commons Public Domain Dedication waiver (http://creativecommons.org/publicdomain/zero/1.0/) applies to the data made available in this article, unless otherwise stated. 


\section{Background}

Patient-reported outcome measures (PROMs) are standardised and validated questionnaires developed for the purpose of gathering outcome data from the patient's perspective [1]. PROMs have come to play an increasingly important role in both clinical practice and research [2-5]. In the present clinical and research climate there are several generic (health condition non-specific) health-related quality-of-life measures available such as the EuroQol 5D (EQ-5D) [6] or the Short Form 36 (SF-36) [7]. These quality-of-life PROMs are currently used regularly in research studies to evaluate the effectiveness of interventions post stroke [8] and are integrated into healthcare systems for use in clinical settings [1].

However, there is currently no conclusive evidence regarding the best method of administration of a PROM with stroke survivors. Potential methods of administration include face-to-face, telephone, online and via the post. This choice of comparators is supported by the findings of the Sentinel Stroke National Audit Programme (SSNAP) [9], which found that all four methods of administration are utilised for 6-month post-stroke follow-ups, with face-to-face follow-up being the most common method.

PROMs response rates post stroke are mostly reported as secondary outcomes, in studies which concentrate on either the development of new PROMs or the reliability and/or validity of PROMs in specific conditions. In those studies that report response rate as a primary outcome, a large variability exists in the response rates reported from 70.1\% [10] for a study by Lannin et al. (2013) comparing the cost-effectiveness of telephone vs mail methods of follow-up for the Australian National Stroke Registry to $45 \%$ [11] for a study by Duncan et al. (2005) examining response rate for the Stroke Impact Scale [12] when administered via mail or telephone methods in veterans. Recently, a cohort study conducted in the London area by Peters et al. (2014) examining PROMs collection by post, in primary care, for those with a long-term condition, received a response rate of $36.4 \%$ [13] for stroke survivors. Thus, with large levels of variability in response rates of previous studies this study has opted for a pragmatic design across a large number of research sites covering rural and urban populations.

The PROM to be utilised in this study is the Patient-reported Outcomes Measurement Information System 10 questions (PROMIS-10 Global Health) [14]. This PROM is recommended by the International Consortium of Health Outcomes Measurement (ICHOM) as part of a proposed standard set of minimum outcome data for stroke survivors developed by a global team of stroke specialists $[15,16]$. However, the Stroke Standard Set working group proposes the inclusion of extra patient-facing health status questions. The first addition are three questions relating to ambulation, toileting and dressing that have been adopted from the RiksStroke (The National Quality Register for Stroke - Sweden) $[17,18]$. Additionally, two further questions on feeding and communication were developed [15] by the ICHOM working group for stroke for simple yes/no responses. The 10 question in PROMIS-10 and separately the three questions from the RiksStroke and two questions from the ICHOM Stroke Standard Set will be utilised for this study. This group of 15 questions will, for ease of reference, be referred to as Patient-reported Health Status Questions (PRHSQs).

Therefore, the objective of this research study is to provide an evidence base for the acceptability of modes of administration of the PROMIS-10 and five additional questions for stroke survivors, and to evaluate if online, postal and telephone modes are not inferior to face-to-face administration.

\section{Methods/design \\ Study design}

This study is a UK four- arm, pragmatic, multicentre, non-inferiority randomised controlled trial of the method of administering the PRHSQs at the 6-month post-stroke time point (clinically confirmed stroke diagnosis $\geq 4$ months to $\leq 8$ months). The ratio of allocation is $1: 1: 1: 1$, with face-to-face being the method of administration against which the non-inferiority of the three comparator methods (online, postal and telephone) will be discerned. Participants will be randomly allocated to one of four methods of administering these 15 Patient-reported Health Status Questions at eligibility and asked to consent via the post. By virtue of the fact that any of the four methods under investigation are established practices in UK stroke services, participants will not be informed about the other possible modes of administration. Addressing this research question requires implementation of a Zelen design [19] this acknowledges the issues of contacting patients prior to obtaining consent. The impact of this design is due to the nature of the intervention, approaching participants later than usual, as well as a larger-than-usual sample size. We acknowledge that informing participants of the alternative methods of administration may introduce bias, as the rate of return of the PRHSQs for the different assessment methods is the primary outcome of interest. Therefore, participants will knowingly consent to receive and complete the PRHSQs via a single method of administration. See Fig. 1 for the participant flow diagram The study protocol was written in full compliance with the Standard Protocol Items: Recommendations for Interventional Trials (SPIRIT) 2013 [20] and a completed SPIRIT Checklist and Figure 2013 (Fig. 2) [21] are available as a supplement (Additional file 1). 


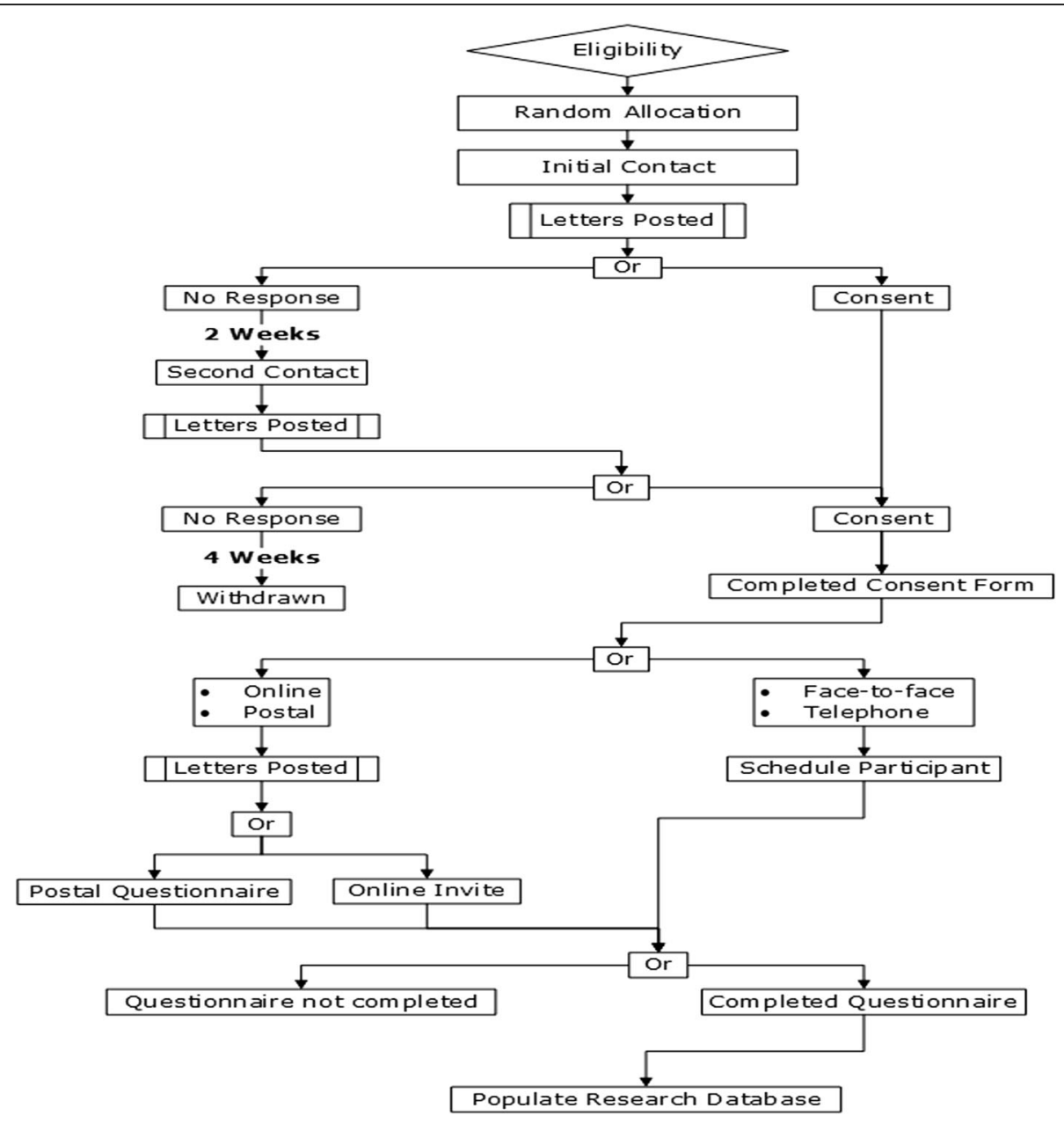

Fig. 1 Participant flow diagram

\section{Recruitment}

The study has gained acceptance by the Health and Care Research Wales (HCRW) Clinical Research Portfolio, thereby allowing for National Health Service (NHS) organisations in all four devolved nations, via the UK Clinical Research Network Portfolio of studies, to view the basic study design and to approach the central research team for permission to open as a research site and conduct the research. The researchers will conduct an open-door policy and will offer the study to any capable NHS organisation who expresses an interest, based on the following site inclusion criteria: (1) patients who meet the study inclusion criteria are routinely under the care of the principle investigator (PI) at the research site and (2) the research site is able to facilitate all four arms of the study.

\section{Study setting}

The study will be undertaken in 14 centres across England and Wales. Centres acting as research sites will be those who were previously involved in potentially eligible participants' post stroke care. In line with the time-frame of the study, the majority of potentially eligible participants will reside in the community and will be anticipating routine clinical follow-up from the centres acting as research sites.

\section{Study population; inclusion and exclusion criteria - Inclusion}

- Clinically confirmed diagnosis of stroke either Cerebral Infarct (ICD I63), Cerebrovascular Haemorrhage (ICD I61) or Stroke, not specified as haemorrhage or infarction (ICD I64) [22]

- Patients aged $\geq 18$ years

- Received a clinically confirmed diagnosis of stroke within the last 4-8 months (stroke diagnosis $\geq$ 4 months to $\leq 8$ months)

\section{Exclusion}

- Clinically confirmed diagnosis of a Transient Ischaemic Attack (ICD G45) [22] 


\begin{tabular}{|c|c|c|c|c|}
\hline & \multicolumn{4}{|c|}{ STUDY PERIOD } \\
\hline & Enrolment & Allocation & Consent & Post-allocation \\
\hline TIMEPOINT & $-t_{1}$ & 0 & $-t_{2}$ & $t_{1}$ \\
\hline \multicolumn{5}{|l|}{ ENROLMENT: } \\
\hline Eligibility screen & $\mathrm{x}$ & & & \\
\hline Informed consent & & & $x$ & \\
\hline Allocation & & $\mathrm{x}$ & & \\
\hline \multicolumn{5}{|l|}{$\begin{array}{l}\text { INTERVENTIONS: PROMIS-10 (Global Health), } \\
\text { RiksStroke } 3 \text { Questions \& ICHOM Stroke } \\
\text { Standard Set } 2 \text { Questions [15 Questions in } \\
\text { Total] }\end{array}$} \\
\hline [Postal Questionnaire] & & & & $x$ \\
\hline [Online Questionnaire] & & & & $\mathrm{x}$ \\
\hline [Face-to-face Questionnaire] & & & & $\mathrm{x}$ \\
\hline [Telephone Questionnaire] & & & & $\mathrm{x}$ \\
\hline \multicolumn{5}{|l|}{ ASSESSMENTS: } \\
\hline $\begin{array}{l}\text { [Demographic Data - Date of Birth, Sex, Date } \\
\text { of Diagnosis and District level Postcode] }\end{array}$ & $x$ & & & \\
\hline $\begin{array}{l}\text { [Health Related Demographic Data - Individual } \\
\text { National Institutes of Health Stroke Score } \\
\text { (NIHSS) on admission, Classification of } \\
\text { Stroke (i.e. Infarct or Haemorrhage using ICD } \\
\text { classifications), Modified Rankin Score on } \\
\text { discharge, whether the participant received } \\
\text { Thrombolysis Yes/No and whether or not the } \\
\text { participant has been diagnosed with Aphasia] }\end{array}$ & $\mathrm{x}$ & & & \\
\hline
\end{tabular}

Fig. 2 Standard Protocol Items: Recommendations for Interventional Trials (SPIRIT) Figure

- Clinically confirmed diagnosis of a Subarachnoid Haemorrhage (ICD I60) [22]

\section{Sample size}

Using a non-inferiority margin of $2.5 \%$, with a power of $80 \%$ and $2.5 \%$ significance level and expecting the same response rate in each group, a minimum of 202 patients are required per randomised allocation group. Thus, a minimum total of 808 randomised eligible participants are necessary for statistical analysis. No adjustment will be made for carrying out three pair-wise tests. Secondary outcomes may not necessarily be powered.

\section{Randomisation}

The sequence was generated using a varying-sized permuted block design, stratified within centre. The sequence was concealed by the central research team on a secure system. The allocation sequences are concealed from analyst and central research team until planned unblinding following interpretation of the study findings. Participants and research staff at the research sites will not be blinded; however, allocation will be random as to not introduce bias.

Potentially eligible participants are randomised and allocated centrally by the research team via e-mail (sent within a secure e-mail network) for the random allocation of potentially eligible participants identified solely via a completely pseudonymous identifier (10-digit NHS Number). Research sites will then receive the 10-digit pseudonymous identifier, a 6-digit Participant Research Number (the first two digits being a site identifier and the subsequent four digits referring to the participant) and the method assigned to. The use of a pseudonymous participant research number is to aid compliance with data governance and to ensure blinding of the participants to the central research team.

\section{Trial procedure}

Potentially eligible participants who have been found eligible by a member of their own clinical care team acting as principal investigator (PI) at the research site, will firstly be randomly allocated to each of the four arms of the study. Following random allocation, potentially eligible participants will be asked to consent by the PI to the research study based on the method of administration they have been randomly allocated. The potentially eligible participants will receive an Invitation Letter, a Participant Information Sheet and a Consent Form via the post. The participant facing documentation will invite the participant to consent to the study and receive their 6-month review and the PRHSQs via one of the four methods they have previously been randomly 
allocated. Additionally, a proxy consent option is noted in the Participant Information Sheet and Consent Form, thereby allowing for a designee to offer consent on behalf of the participant via the principles of informed consent if the participant requires physical assistance to complete the questionnaire.

Following the initial contact, participants who have not responded will be contacted a second time; two working weeks following the dispatch of the first letter. The second contact will contain the same information as the initial contact (Invitation Letter, Participant Information Sheet and Consent Form). Potentially eligible participants will be given a further four working weeks to reply to the second invitation to participate. If no consent is returned, the potentially eligible participant will be assumed not to wish to take part and will not be asked to complete the PRHSQs.

For all potentially eligible participants who are randomised and invited to participate in the study, we will collect a range of background demographic data and a small number of routinely collected health-related data. See Table 1 for details of data for collection.

All demographic data and routinely collected clinical data will be stored against a Participant Research Number in the research data base. Personally identifiable information will only be stored at the research sites and will not be stored for those who do not offer informed their consent before the cut-off period of 4 weeks following the posting of the second letter Therefore, no personally identifiable information will be held by the central research team.

All consented participants will receive the PRHSQs. The PI at site as a member of both the research team and the participant's own care team will be responsible for arranging or delegating the completion of the questionnaires between 4 and 8 months post stroke. There are schema for each method by which the PI must abide; however, the methodologies for all four methods of administration differ slightly and are as follows:

\section{Face-to-face interview}

As part of their routine care, the consented participants will receive a 6-month post-diagnosis review appointment with either a clinical nurse specialist (CNS) in stroke or their clinician. During the 6-month post-stroke review appointment the PRHSQ will be administered by a member of the research team. If participants fail to attend the designated appointment they will not be contacted as part of the study but may potentially be followed up by their own care team at a later date.

\section{Telephone interview}

The consented participants will receive through the post an appointment time to receive a telephone interview for the 6-month post-diagnosis review appointment with either a CNS in stroke or their clinician. During the 6-month post-stroke review appointment the PRHSQ will be administered by a member of the research team. If participants fail to meet the agreed appointment, they will not be contacted again by the research team, but may potentially be followed up by their own care team at a later date.

\section{Postal questionnaire}

The consented participants will receive a paper version of the PRHSQ. In addition, participants will receive a pre-paid envelope to return it to the research team. The questionnaire will be answered and filled in by either the participant or designated proxy if the participant is unable to physically complete the questionnaire. If participants fail to respond, they will not be contacted again by the research team, but may potentially be followed up by their own care team at a later date.

Table 1 Demographic and routine clinical data for collection

\begin{tabular}{|c|c|}
\hline Demographic data & Routinely collected clinical data \\
\hline $\begin{array}{l}\text { Date of birth } \\
\text { DD/MM/YYYY }\end{array}$ & $\begin{array}{l}\text { Classification of stroke: } \\
\text { Cerebral Infarct (ICD I63) } \\
\text { Cerebrovascular Haemorrhage (ICD I61) } \\
\text { Stroke - not specified as Haemorrhage or Infarction (ICD I64) }\end{array}$ \\
\hline \multirow{4}{*}{$\begin{array}{l}\text { Sex } \\
\text { District-level postcode - } \\
\text { First 3-4 digits of a } \\
\text { UK post code }\end{array}$} & $\begin{array}{l}\text { Date of index event (date of stroke) } \\
\text { Individual National Institutes of Health Stroke Score (NIHSS) on admission: } \\
0-42\end{array}$ \\
\hline & $\begin{array}{l}\text { Treated with thrombolysis? } \\
\text { Yes / No }\end{array}$ \\
\hline & $\begin{array}{l}\text { Modified Rankin Score on discharge or transfer from stroke unit: } \\
0-6\end{array}$ \\
\hline & $\begin{array}{l}\text { Clinically confirmed diagnosis of aphasia as sequela of ICD 163, } 161 \text { or } 164 \text { ?: } \\
\text { (data collected at } 3 \text { sites within a single Health Board only) } \\
\text { Yes / No }\end{array}$ \\
\hline
\end{tabular}




\section{Online questionnaire}

The consented participants will receive a postal invitation to access a secure online version of the PRHSQ via a secure web address (Bristol Online Survey - https:// www.onlinesurveys.ac.uk/).. The online questionnaire will be answered and filled in by either the participant or designated proxy if the participant is unable to physically complete the questionnaire. If participants fail to complete the online questionnaire, they will not be contacted again by the research team, but may potentially be followed up by their own care team at a later date.

\section{Data management}

Data will be collected at the research sites until February 2018. Potentially eligible participants who do not respond or consent will not be excluded from data analysis of their demographic and routinely collected non-identifiable clinical data as this data is essential for the determination of acceptability of the methods under investigation. However, individuals who do not consent will not receive the PRHSQs.

Two levels of data management exist for this research study. The first level is that of the individual research site which will have access to identifiable patient data. This will allow the site to invite potentially eligible participants and to contact participants who consent to receive the PRHSQs via the method randomly assigned. Following completion of the PRHSQs the anonymous data received from the two self-administered questionnaires (postal or online) will be populated in the research data base against the corresponding Participant Research Number as detailed on the returned PRHSQs. The data from the two administered methods (face-to-face or telephone) will be captured via anonymised PRHSQs, identified solely by the Participant Research Number.. Data is to be initially coded and entered into a site level data base. The data base has been developed using Microsoft Excel 2013 to support 'data validation' and will not accept any data entered outside pre-defined ranges for each discrete data value to be collected. Additionally, 'conditional formatting' will be utilised to ensure that double entry is minimised..

The second level of data management is that of the central research team. No personally identifiable information will be transferred or held in the central research data base. Research sites will transfer data to the research team using the Participant Research Number as the unique discriminator. Data quality will be ensured by the trial statistician (Dr. Ben Carter, Senior Lecturer in Biostatistics, King's College London) by conducting central data verification prior to data base lock. Data queries arising from data verification procedures will be submitted to sites in writing and sites will have two working weeks to respond in full.
All data will be handled by research sites and by the central research team in accordance with the Data Protection Act (1998) [23]. Subsequent to the closure of the study all trial materials will be archived for a period defined by Good Clinical Practice (ICH-GCP). The study will be conducted in accordance with the Declaration of Helsinki.

\section{Monitoring}

Site monitoring visits will occur throughout the conduct of the study. These monitoring visits will be conducted by members of the central research team and will ensure the correct conduct of the study and will identify any protocol deviations. Furthermore, site monitoring visits will support sites and will offer the opportunity for the central research team to improve the conduct of the study by identifying needs at the research site and offer any clarification or training necessary. The principal investigators (PIs) at research sites are responsible for monitoring protocol deviations and sites are required to report all deviations via the protocol deviation form. All deviations will be monitored and assessed by the central research team. Following assessment, corrective and preventative actions will be disseminated to the sites.

\section{Adverse events}

All harms will be reported utilising an adverse and serious adverse event (AE, SAE) form included in the research site file, which will be disseminated to all research sites during site initiation visits. We do not anticipate any trial-related AEs; however, sites will collect and report all AEs and SAEs to the central research team within $24 \mathrm{~h}$ of discovery as per the Health and Care Research Wales guidelines. In the event of a trial-related $\mathrm{AE}$ or SAE the research site will escalate this to their local NHS R\&D department, National Research Governance Organisation and to the chief investigator (CI). Additionally, all PRHSQs can be unblinded via the research sites in the rare event that a concern for patient safety or the safety of others is identified.

\section{Outcomes \\ Primary outcome}

- The proportion of individuals eligible for the study who return the method specific PRHSQs in each of the four study arms (postal, online, face-to-face and telephone)

\section{Secondary outcomes}

- The proportion of individuals eligible for the study who return the method specific PRHSQs in each of the four study arms (postal, online, face-to-face and telephone) for individuals with communication issues (i.e. aphasia) 
- The proportion of individuals eligible for the study who return the method specific PRHSQs in each of the four study arms (postal, online, face-to-face and telephone) by stroke severity as defined by individual NIHSS on admission

- The proportion of individuals eligible for the study who return the method-specific PRHSQs in each of the four study arms (postal, online, face-to-face and telephone) by stroke type; Cerebral Infarct (ICD I63), Cerebrovascular Haemorrhage (ICD 161) or Stroke, not specified as haemorrhage or infarction (ICD I64)

\section{Statistical analysis}

\section{Analysis of the primary outcome}

We will carry out a difference of two proportions between the three intervention groups (postal, telephone or online) verses face-to-face administration, with a $2.5 \%$ non-inferiority margin. The three comparisons will be adjusted using a Holm [24] multiplicity adjustment, with a two-sided alpha $=0.05$. The analysis will be presented as a difference with an associated 95\% confidence interval (CI).

Analyst and researchers blinding to the allocation will remain in place until interpretation of the primary and secondary outcomes are agreed. Following un-blinding of the allocation, difference will be generated in the three comparator allocation groups, and face-to-face completion rate, and will include the non-inferiority margin of $2.5 \%$, as our primary analysis.

\section{Secondary analysis of the primary outcome}

We plan to repeat the primary analysis after adjustment for: age; sex; National Institutes of Health Stroke Score (NIHSS) on admission and stroke type (Haemorrhagic or Ischaemic).

\section{Analysis of the secondary outcomes}

Continuous data will be summarised descriptively, and also with a general linear model, and adjusted for patient age and sex. Dichotomous data will be summarised descriptively and will analysed using a logistic regression adjusted for: patient age and sex.

We note that many data will be gathered relating to the questionnaire itself. This useful clinical data warrants publication in its own right; however, it is not the focus of this study and will form a separate stand-alone analysis.

\section{Missing data and analysis population}

We will be reporting using a complete case analysis. Due to the nature of the research question, we are expecting missing data, and anticipate that this will be not missing at random. We will describe patterns of missing data. However, the primary objective of this study is to ascertain if the missing data is systematically more likely in the comparator groups, compared to the face-to-face allocation group.

\section{Non-inferiority margin}

Utilising unpublished routinely collected clinical data approximately $85 \%$ of follow-up appointments for all patients, who would meet the inclusion criteria, return for face-to-face administration of their 6-month post-stroke follow-up assessment at the $\geq 4$-month to $\leq 8$-months post-diagnosis time point. The reason for this high level of face-to-face follow-up is not grounded on evidence, however, disability and communication issues post stroke may have a role in the choice of this method. Nevertheless, there is a significant cost implication in the choice of method for follow-up as evidenced in a study by Lannin et al. (2013) investigating the cost-effectiveness of telephone vs mail methods of follow-up for the Australian National Stroke Registry [10].

Alongside the National Institute for Health and Care Excellence (NICE) accredited RCP stroke guidelines [25], a non-inferiority margin of $2.5 \%$ was decided upon as the maximum tolerated acceptable reduction in acceptability, alongside conflicting resource allocation and offering flexibility of patients to undergo follow-up.

\section{Trial governance}

\section{Trial management group}

The study has been delivered to the highest standard overseen by the Trial Management Group (TMG). The TMG met routinely to develop and approve the following: trial documents (Participant Information Sheet, Invitation Letter, and Protocol), Randomisation Protocol; trial database; Case Report Forms. The TMG will be convened routinely to discuss trial-related issues as they arise (e.g. protocol deviations, AEs and SAEs); changes to the protocol; data management plan and statistical analysis plan.

\section{Trial Steering Committee}

The study is to be overseen by a Steering Committee composed of Dr. Manju Krishnan (Clinical Lead and Consultant Stroke Physician Abertawe Bro Morgannwg University Health Board and Deputy HCRW Stroke Research Lead) as chair, lay members, independent clinical and research specialists and key stakeholders. The Steering Committee will be responsible for examining research progress, monitoring progress against established timelines and monitoring recruitment, completion and follow-up rates. Meetings will take place quarterly and all recommendations arising from the Steering Committee will be acted upon immediately. Moreover, the Steering Committee will reserve the right to request and receive interim reports between quarterly meetings. The nature and design of the trial does not necessitate the creation of an additional Data Management 
Committee to supplement the work of the Trial Steering Committee. Therefore, the Trial Steering Committee will have oversight across the study, including data monitoring and data management.

\section{Discussion}

This pragmatic randomised controlled trial will provide insight into the optimal method of PROM delivery in stroke survivors and also inform the choice of delivery method across other chronic disease conditions, especially conditions associated with chronic physical disability. The choice of a pragmatic study design, whilst offering good generalisability and the testing of a hypothesis under as near to 'real-world' conditions as is possible, can and will offer substantial challenges. The 'natural' organisational variances of each research site, require careful and thorough discussions to ensure that research is not disruptive to routine clinical work and that the research procedures outlined within the protocol are adhered to.

We assume a possibility that the pragmatic design of the research study will not be accessible to all individuals post stoke. Nevertheless, all eligible individuals are to be accounted for in data analysis. It is believed, that this will offer possible correlations as to why certain demographic and clinical features relate to the number of consented participants for each allocated method.

The benefits of this study, to stroke survivors is in the fact that they will offer clinicians and healthcare commissioners early evidence as to which method they find most acceptable, when compared against the most commonly commissioned approach. Moreover, due to the assumption that data is missing not at random, those who do not respond offer useful data. Therefore, any actions taken on the basis of the final analysis of this study can do so in the knowledge that those actions are based on the preferences of all the eligible stroke survivors approached for the study.

\section{Dissemination}

We plan to disseminate the findings of the study via a peer-reviewed academic journal. The findings of which will be cascaded to all research sites and disseminated from the research sites to participants. The published findings will adhere to the Consolidated Standards of Reporting Trials (CONSORT) randomised control trial reporting guidelines $[26,27]$. In addition, the published findings will adhere to both the CONSORT extension relating to non-inferiority and equivalence trials [28] and the extension relating to pragmatic trials [29].

\section{Trial status}

Recruitment commenced in July 2017 and is planned to continue until February 2018. Protocol Version: 1.0 Date: 10 April 2017.

\section{Additional file}

Additional file 1: Standard Protocol Items: Recommendations for Interventional Trials (SPIRIT) 2013 Checklist: recommended items to address in a clinical trial protocol and related documents. (DOC $121 \mathrm{~kb}$ )

\section{Abbreviations}

CNS: Clinical nurse specialist; CONSORT: Consolidated Standards of Reporting Trials; EQ-5D: EuroQol 5 Dimensions; GCP: Good Clinical Practice;

HCRW: Health and Care Research Wales; ICD: International Classification of Diseases; ICHOM: International Consortium for Health Outcomes

Measurement; NHS: National Health Service; NICE: National Institute for Health and Care Excellence; NIHSS: National Institutes of Health Stroke Score; PI: Principle investigator; PRHSQs: Patient-reported Health Status Questions; PROM/s: Patient-reported outcome measure/s; PROMIS-10: Patient-reported Outcomes Measurement Information System 10 questions; RCP: Royal College of Physicians; SF-36: Short Form 36; SPIRIT: Standard Protocol Items: Recommendations for Interventional Trials; SSNAP: Stroke Sentinel Audit Programme; URL: Uniform Resource Locator (colloquially referred to as a 'web address')

\section{Acknowledgements}

The authors would like to acknowledge the work of the Steering Committee and in particular John and Karen Soos and Dr. Manju Krishnan.

\section{Funding}

This research study was funded by a competitive research grant awarded by the Stroke Implementation Group (SIG) for Wales. The SIG is funded directly by Welsh Government to address the needs of stroke survivors in Wales. The funder had no role in the design of this study and will not have any role during its execution, analyses, interpretation of the data, nor decision to submit results. This study represents independent research part funded by the National Institute for Health Research (NIHR) Biomedical Research Centre at South London and Maudsley NHS Foundation Trust and King's College London (BRC). The views expressed are those of the authors and not necessarily those of the NHS, the NIHR or the Department of Health and Social Care.

\section{Availability of data and materials}

The final dataset will be accessible only by the authors. The authors reserve the rights to utilise the final dataset for discreet secondary analyses separate from the main trial analyses.

\section{Authors' contributions}

$\mathrm{JH}$ and BC conceived and developed the study. SG, MP, RD and JW helped to develop the study. AS aided in study conception, led in the study design and protocol development. AP led the implementation of the study with guidance from $\mathrm{AS}, \mathrm{JH}$ and $\mathrm{BC}$. BC acted as the blinded trial statistician and provided statistical expertise for the trial design and will conduct the primary data analysis. AS, BC and $\mathrm{JH}$ contributed to the refinement of the study protocol and approved the final manuscript. All authors read and approved the final manuscript.

\section{Ethics approval and consent to participate}

Ethical approval was obtained from the North West - Greater Manchester South Research Ethics Committee via the Health Research Authority (HRA) Central Booking Service as a member panel of the NHS Research Ethics Committees (IRAS ID - 222,226 - REC ID - 17/NW/0269). All potentially eligible participants will be approached to offer their informed consent.

\section{Competing interests}

The authors declare that they have no competing interests.

\section{Publisher's Note}

Springer Nature remains neutral with regard to jurisdictional claims in published maps and institutional affiliations.

\section{Author details}

${ }^{1}$ Aneurin Bevan University Health Board, South Wales, UK. ${ }^{2}$ Department of Biostatistics and Health Informatics, Institute of Psychiatry, Psychology \& 
Neuroscience, King's College London, London, UK. ${ }^{3}$ Powys Teaching Health Board, Mid Wales, UK. ${ }^{4} \mathrm{C}$ wm Taf University Health Board, South Wales, UK.

${ }^{5}$ Division of Population Medicine, Cardiff University, Cardiff, UK.

Received: 1 December 2017 Accepted: 17 May 2018

Published online: 03 July 2018

\section{References}

1. Dawson J, Doll H, Fitzpatrick R, Jenkinson C, Carr AJ. The routine use of patient reported outcome measures in healthcare settings. BMJ. 2010;340: c186. https://doi.org/10.1136/bmj.c186

2. Greenhalgh J. The applications of PROs in clinical practice: what are they, do they work, and why? Qual Life Res. 2009;18:115-23. https://doi.org/10. 1007/s11136-008-9430-6

3. Snyder CF, Aaronson NK, Choucair AK, Elliott TE, Greenhalgh J, Halyard MY, et al. Implementing patient-reported outcomes assessment in clinical practice: a review of the options and considerations. Qual Life Res. 2012;21: 1305-14. https://doi.org/10.1007/s11136-011-0054-x

4. Higginson IJ, Carr AJ. Measuring quality of life: using quality of life measures in the clinical setting. BMJ. 2001;322:1297-300. https://doi.org/10.1136/bmj. 322.7297.1297

5. Alonso J, Bartlett SJ, Rose M, Aaronson NK, Chaplin JE, Efficace F, Leplège A, Lu A, Tulsky DS, Raat H, Ravens-Sieberer U, Revicki D, Terwee CB, Valderas JM, Cella D, Forrest CB. The case for an international patient-reported outcomes measurement information system (PROMIS ${ }^{\oplus}$ ) initiative Health Qual Life Outcomes. 2013;11(1), p. 210. doi: https://doi.org/10.1186/1477-7525-11-210.

6. Devlin NJ, Brooks R. EQ-5D and the EuroQol group: past, present and future. Appl Health Econ Health Policy. 2017:1-11. https://doi.org/10.1007/s40258017-0310-5.

7. Hays RD, Sherbourne CD, Mazel RM. The Rand 36-item health survey 1.0. Health Econ. 1993;2(3):217-27. https://doi.org/10.1002/hec.4730020305.

8. Cogger $\mathrm{H}$, Worthington $\mathrm{E}$, Lincoln NB. Cognitive rehabilitation for memory deficits after stroke. Cochrane Database Syst Rev. 2016; https://doi.org/10. 1002/14651858.cd002293.pub3.

9. Sentinel Stroke National Audit Programme (SSNAP). SSNAP Portfolio for April-July 2016 admissions and discharges - Wales.2016. [Online] Available at: https://www.strokeaudit.org/Documents/GroupType/NHSW/NHSW01/ AprJul2016/NHSW01-AprJul2016-ResultsPortfolio.aspx. Accessed 5 Apr 2017.

10. Lannin N, Anderson C, Lim J, Paice K, Price C, Faux S, Levi C, Donnan G, Cadilhac D. Telephone follow-up was more expensive but more efficient than postal in a national stroke registry. J Clin Epidemiol. 2013. 66(8):896-902.

11. Duncan P, Reker D, Kwon S, Lai S, Studenski S, Perera S, Alfrey C, Marquez J. Measuring stroke impact with the stroke impact scale. Med Care. 2005;43(5): 507-15.

12. Duncan P, Bode R, Min Lai S, Perera S. Rasch analysis of a new strokespecific outcome scale: the Stroke Impact Scale. Arch Phys Med Rehabil. 2003;84(7):950-63.

13. Peters $M$, Crocker $H$, Jenkinson C, Doll H, Fitzpatrick R. The routine collection of patient-reported outcome measures (PROMs) for long-term conditions in primary care: a cohort survey. BMJ Open. 2014;4(2):e003968. https://doi.org/ 10.1136/bmjopen-2013-003968

14. Cella D, Riley W, Stone A, Rothrock N, Reeve B, Yount S, Amtmann D, Bode R, Buysse D, Choi S, Cook K, DeVellis R, DeWalt D, Fries JF, Gershon R, Hahn EA, Lai J-S, Pilkonis P, Revicki D, Rose M, Weinfurt K, Hays R. The patientreported outcomes measurement information system (PROMIS) developed and tested its first wave of adult self-reported health outcome item banks: 2005-2008. J Clin Epidemiol. 2010;63(11):1179-94. https://doi.org/10.1016/j. jclinepi.2010.04.011.

15. International Consortium for Health Outcomes Measurement (ICHOM) Stroke: Data Collection Reference Guide - Version 2.0. 2015.

16. Salinas J, Sprinkhuizen SM, Ackerson T, Bernhardt J, Davie C, George MG Gething S, Kelly AG, Lindsay P, Liu L, Martins SCO, Morgan L, Norrving B, Ribbers GM, Silver FL, Smith EE, Williams LS, Schwamm LH. An international standard set of patient-centered outcome measures after stroke. Stroke. 2015:47(1):180-6. https://doi.org/10.1161/strokeaha.115.010898.

17. Asplund K, Hulter Åsberg K, Appelros P, Bjarne D, Eriksson M, Johansson $\AA$, Jonsson F, Norrving B, Stegmayr B, Terént A, Wallin S, Wester PO. The RiksStroke story: building a sustainable national register for quality assessment of stroke care. Int J Stroke. 2011;6(2):99-108. https://doi.org/10.1111/j.17474949.2010.00557.x
18. Söderholm A, Stegmayr B, Glader E-L, Asplund K. Validation of hospital performance measures of acute stroke care quality. RiksStroke, the Swedish Stroke Register. Neuroepidemiology. 2016;46(4):229-34. https://doi.org/10. 1159/000444679.

19. Adamson J, Cockayne S, Puffer S, Torgerson DJ. Review of randomised trials using the post-randomised consent (Zelen's) design. Contemp Clin Trials. 2006;27(4):305-19. https://doi.org/10.1016/j.cct.2005.11.003.

20. Chan A-W, Tetzlaff JM, Gøtzsche PC, Altman DG, Mann H, Berlin J, Dickersin K, Hróbjartsson A, Schulz KF, Parulekar WR, Krleža-Jerić K, Laupacis A, Moher D. SPIRIT 2013 explanation and elaboration: guidance for protocols of clinical trials. BMJ. 2013;346:e7586.

21. Chan A-W, Tetzlaff JM, Altman DG, Laupacis A, Gøtzsche PC, Krleža-Jerić K, Hróbjartsson A, Mann H, Dickersin K, Berlin J, Doré C, Parulekar W, Summerskill W, Groves T, Schulz K, Sox H, Rockhold FW, Rennie D, Moher D. SPIRIT 2013 statement: defining standard protocol items for clinical trials. Ann Intern Med. 2013;158:200-7.

22. World Health Organization. The ICD-10 classification of mental and behavioural disorders: clinical descriptions and diagnostic guidelines. Geneva: World Health Organization; 1992.

23. Great Britain Data Protection Act. London: Stationery Office [Online] 1998. Available at: http://www.legislation.gov.uk/ukpga/1998/29/contents Accessed 2 Mar 2017.

24. Holm S. A simple sequentially rejective multiple test procedure. Scand J Stat. 1979;6(2):65-70.

25. Intercollegiate Stroke Working Party. National clinical guideline for stroke. 5th ed. London: Royal College of Physicians; 2016.

26. Schulz KF, Altman DG, Moher D, for the CONSORT Group. CONSORT 2010 statement: updated guidelines for reporting parallel group randomised trials. Ann Int Med. 2010;152 Epub 24 March

27. Moher D, Hopewell S, Schulz KF, Montori V, Gøtzsche PC, Devereaux PJ, Elbourne D, Egger M, Altman DG. CONSORT 2010 explanation and elaboration: updated guidelines for reporting parallel group randomised trials. J Clin Epi. 2010:63(8):e1-e37.

28. Piaggio G, Elbourne DR, Pocock SJ, Evans SJW, Altman DG, for the CONSORT group. Reporting of noninferiority and equivalence randomized trials. Extension of the CONSORT 2010 statement. JAMA. 2012;308(24):2594604. https://doi.org/10.1001/jama.2012.87802. PMID: 23268518.

29. Zwarenstein M, Treweek S, Gagnier JJ, Altman DG, Tunis S, Haynes B, Oxman AD, Moher D, for the CONSORT and Pragmatic Trials in Healthcare (Practihc) Group. Improving the reporting of pragmatic trials: an extension of the CONSORT statement. BMJ. 2008:337:a2390. PMID: 19001484

\section{Ready to submit your research? Choose BMC and benefit from:}

- fast, convenient online submission

- thorough peer review by experienced researchers in your field

- rapid publication on acceptance

- support for research data, including large and complex data types

- gold Open Access which fosters wider collaboration and increased citations

- maximum visibility for your research: over $100 \mathrm{M}$ website views per year

At BMC, research is always in progress.

Learn more biomedcentral.com/submissions 